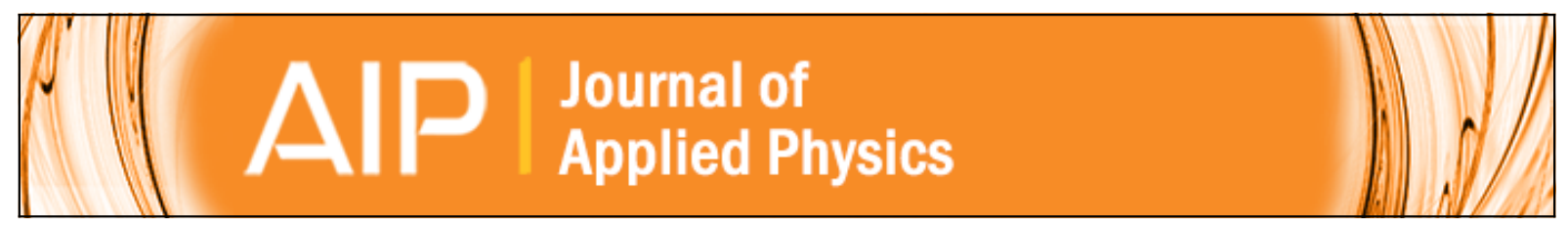

\title{
Design and characterization of broadband magnetoelectric sensor
}

Chee-Sung Park, Cheol-Woo Ahn, Jungho Ryu, Woon-Ha Yoon, Dong-Soo Park, Hyoun-Ee Kim, and Shashank Priya

Citation: Journal of Applied Physics 105, 094111 (2009); doi: 10.1063/1.3117484

View online: http://dx.doi.org/10.1063/1.3117484

View Table of Contents: http://scitation.aip.org/content/aip/journal/jap/105/9?ver=pdfcov

Published by the AIP Publishing

\section{Articles you may be interested in}

Influence of metglas layer on nonlinear magnetoelectric effect for magnetic field detection by frequency modulation

J. Appl. Phys. 117, 024104 (2015); 10.1063/1.4905622

Enhancement in magnetic field sensitivity and reduction in equivalent magnetic noise by magnetoelectric laminate stacks

J. Appl. Phys. 111, 104504 (2012); 10.1063/1.4718441

Thermal stability of magnetoelectric sensors

Appl. Phys. Lett. 100, 173505 (2012); 10.1063/1.4705298

Comparison of noise floor and sensitivity for different magnetoelectric laminates

J. Appl. Phys. 108, 084509 (2010); 10.1063/1.3486483

Geomagnetic sensor based on giant magnetoelectric effect

Appl. Phys. Lett. 91, 123513 (2007); 10.1063/1.2789391

\section{MIT LINCOLN} LABORATORY CAREERS

Discover the satisfaction of innovation and service to the nation
- Space Control

- Air \& Missile Defense

- Communications Systems \& Cyber Security

- Intelligence, Surveillance and

Reconnaissance Systems

\section{LINCOLN LABORATORY}

MassachusetTs Institute OF TeChNOLOGy

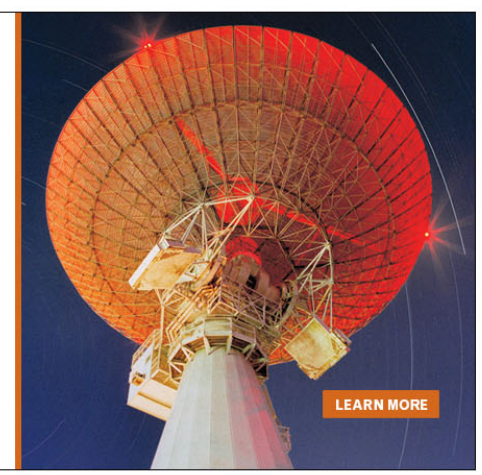




\title{
Design and characterization of broadband magnetoelectric sensor
}

\author{
Chee-Sung Park, ${ }^{1}$ Cheol-Woo Ahn, ${ }^{1}$ Jungho Ryu, ${ }^{2}$ Woon-Ha Yoon, ${ }^{2}$ Dong-Soo Park, ${ }^{2}$ \\ Hyoun-Ee Kim, ${ }^{3}$ and Shashank Priya ${ }^{1, a)}$ \\ ${ }^{1}$ Department of Materials Science and Engineering, Virginia Polytechnic Institute and State University, \\ Blacksburg, Virginia 24061, USA \\ ${ }^{2}$ Functional Ceramics Group, Korea Institute of Materials Science (KIMS), Changwon, Gyeongnam 641- \\ 831, Republic of Korea \\ ${ }^{3}$ Department of Materials Science and Engineering, Seoul National University, Seoul 151-744, Republic of \\ Korea
}

(Received 3 February 2009; accepted 8 March 2009; published online 13 May 2009)

\begin{abstract}
In this study, we present a broadband magnetoelectric (ME) sensor design comprising of Metglas and piezoelectric ceramic laminate composite. A systematic study was conducted to elucidate the role of various composite variables toward the ME response [longitudinal-transverse (LT) mode] over the applied range of magnetic dc bias. The broadband behavior was characterized by flat $\mathrm{ME}$ responses over a wide range of magnetic dc bias at frequency of $1 \mathrm{kHz}$. The variation in $\mathrm{ME}$ coefficient as a function of magnetic dc bias was found to be significantly dependent on the size and shape of the laminate composites, the number of Metglas layers, and composite structure of sandwich versus unimorph. By adjusting these variables, we were able to achieve near-flat $\mathrm{ME}$ response over a magnetic bias range of 90-220 Oe. ME coefficient was also measured as a function of frequency, and at electromechanical resonance the peak value was found to be almost independent of applied magnetic bias in the range of 90-220 Oe. (C) 2009 American Institute of Physics. [DOI: 10.1063/1.3117484]
\end{abstract}

\section{INTRODUCTION}

Magnetoelectric (ME) materials have been projected to find applications in sensors, transducers, actuators, and servomechanism. ${ }^{1-4} \mathrm{ME}$ effect is a product property and has been widely investigated in composite structures consisting of magnetostrictive and piezoelectric materials. In these composites, ME coefficient is dependent on the elastic coupling occurring at the interface of piezoelectric and magnetostrictive phases. Magnetostrictive-piezoelectric laminate composites have shown much higher ME coefficients than that of single-phase materials or particulate composites. ${ }^{4-8}$ Experimental and analytical results on ME laminates have shown relatively large ME output voltage but only limited to the narrow range of dc magnetic bias. This limits their ability to be utilized for ac magnetic field sensing, which desires flat $\mathrm{ME}$ response over a wide dc magnetic bias range. The common modes utilized for laminates are longitudinal-transverse (LT) and longitudinal-longitudinal, where most of the ME composites exhibit a sharp peak with the maxima occurring over specific dc bias magnitude dependent on the material properties. This maxima position occurs at 5 Oe magnetic dc bias for 2-1 composites consisting of Metglas sheets and piezoelectric fibers, which is probably the lowest. ${ }^{9}$ For sintered composites consisting of PZT and ferrite phases, the maximum occurs at the applied dc bias of 400 Oe. ${ }^{10}$ The ME coefficient peaks at the optimum dc bias are generally sharp, which limits the applicability of these composites.

In this study, we investigate the Metglas and lead zirconate titanate-lead zinc niobate (PZNT) based composites to

\footnotetext{
a) Author to whom correspondence should be addressed. Electronic mail: spriya@vt.edu.
}

overcome the problem of limited dc magnetic bias range associated with laminates. The selection of materials is based on the fact that magnetostriction peak in Metglas occurs at low magnetic dc bias magnitude under $100 \mathrm{Oe}$, while the piezoelectric voltage constant $\left(g_{33}\right)$ in PZNT is high in the range of $23.41 \times 10^{-3} \mathrm{~V} \mathrm{~m} / \mathrm{N}$. Investigations by Dong et $a .^{11-13}$ showed that the voltage generated through ME composite structure is given as

$$
\begin{aligned}
V= & \frac{N d_{33, m} g_{33, p}}{N s_{33}^{E}\left(1-k_{33}^{2}\right)+(1-N) s_{33}^{H}} H t \\
& \text { (open circuit voltage), }
\end{aligned}
$$

where $s_{33}{ }^{E}$ and $s_{33}{ }^{H}$ are the elastic compliances for the piezoelectric and magnetostrictive layers, $k_{33}$ is the electromechanical coupling coefficient of the piezoelectric layer, $d_{33, m}$ and $g_{33, p}$ are the longitudinal piezomagnetic and piezoelectric voltage coefficients, and $n$ is the thickness fraction of magnetostrictive layers. This expression is an approximation and requires an additional correction factor to match the magnitude of voltage coefficient measured experimentally. However, it provides an insight into material selection, and based on this expression it can be seen that piezoelectric voltage constants, elastic compliances (mechanical impedance matching), and piezomagnetic constants are the key factors controlling the magnitude of ME coefficients.

\section{EXPERIMENTAL PROCEDURE}

Piezoelectric plates of composition $\mathrm{Pb}\left(\mathrm{Zn}_{1 / 3} \mathrm{Nb}_{2 / 3}\right)_{x}\left(\mathrm{Zr}_{0.5} \mathrm{Ti}_{0.5}\right)_{1-x} \mathrm{O}_{3}$ (PZNT) were synthesized by conventional mixed oxide method. $\mathrm{PbO}, \mathrm{ZrO}_{2}, \mathrm{TiO}_{2}, \mathrm{ZnO}$, and $\mathrm{Nb}_{2} \mathrm{O}_{5}$ powders (all $99.9 \%$ purity from Sigma Aldrich 

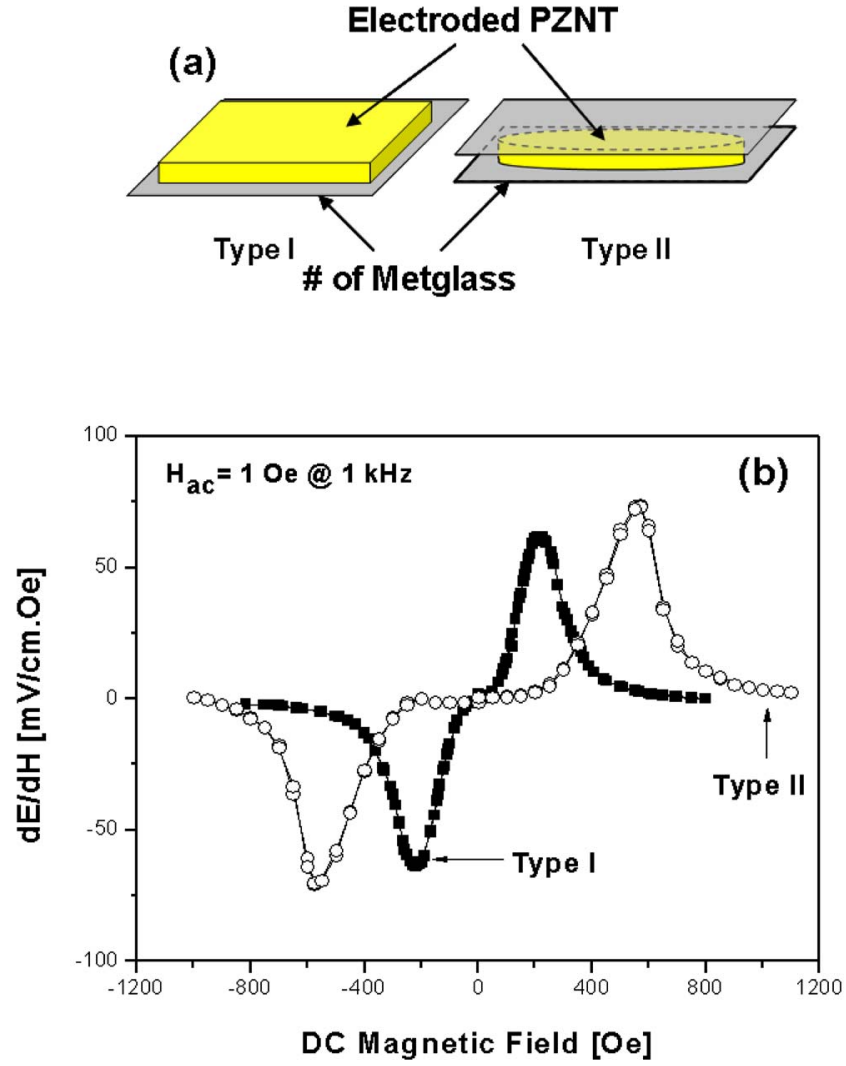

FIG. 1. (Color online) (a) Schematic diagram of fabricated samples. Type I: $15.5 \times 15.5 \mathrm{~mm}^{2}$ PZNT plate with Metglas attached on one side only, and Type II: $10.2 \mathrm{~mm}$ diameter PZNT disk with Metglas attached on both sides. (b) ME output voltage as a function of dc magnetic field.

Co., USA) were weighed and mixed using ball-mill with zirconia balls as media in alcohol. After mixing and drying, the mixture was calcined at $850{ }^{\circ} \mathrm{C}$ for $4 \mathrm{~h}$, and the calcined powder was ball-milled again for $24 \mathrm{~h}$. Dried powders were pressed and sintered at $950{ }^{\circ} \mathrm{C}$ for $4 \mathrm{~h}$ in air. Sintered specimens were machined, electroded using silver paint (DuPont 7713 conductor paste, firing temperature of $650{ }^{\circ} \mathrm{C}$ ), and poled at $4 \mathrm{kV} / \mathrm{mm}$. The piezoelectric constant of poled PZNT plates was found to be $500 \mathrm{pC} / \mathrm{N}$, and dielectric constant was found to be 2219 at $1 \mathrm{kHz}$. On these poled PZNT plates, $25 \mu \mathrm{m}$ thick Metglas (2605SA1, Metglas Inc., USA) sheets of desired dimensions were attached using epoxy (West System, USA) with curing temperature of $80{ }^{\circ} \mathrm{C}$. For the ME voltage coefficient measurement, an electromagnet was used to apply dc magnetic field, and the samples were placed in the center of the Helmholtz coil under an ac magnetic field $\left(H_{\mathrm{ac}}\right)$. ME voltage (LT mode configuration) induced on the laminate was monitored using lock-in amplifier, and impedance spectrum was measured by analyzer (HP4194A, USA). ATILA was used for finite element method (FEM) analysis of resonance modes occurring in the laminate over a wide range of frequency.

\section{RESULTS AND DISCUSSIONS}

\section{A. Effect of shape and laminate configuration}

PZNT square plate and disk were used in this experiment, as schematically depicted in Fig. 1(a). 20 layers of Metglas were stacked on square PZNT plate (15.5
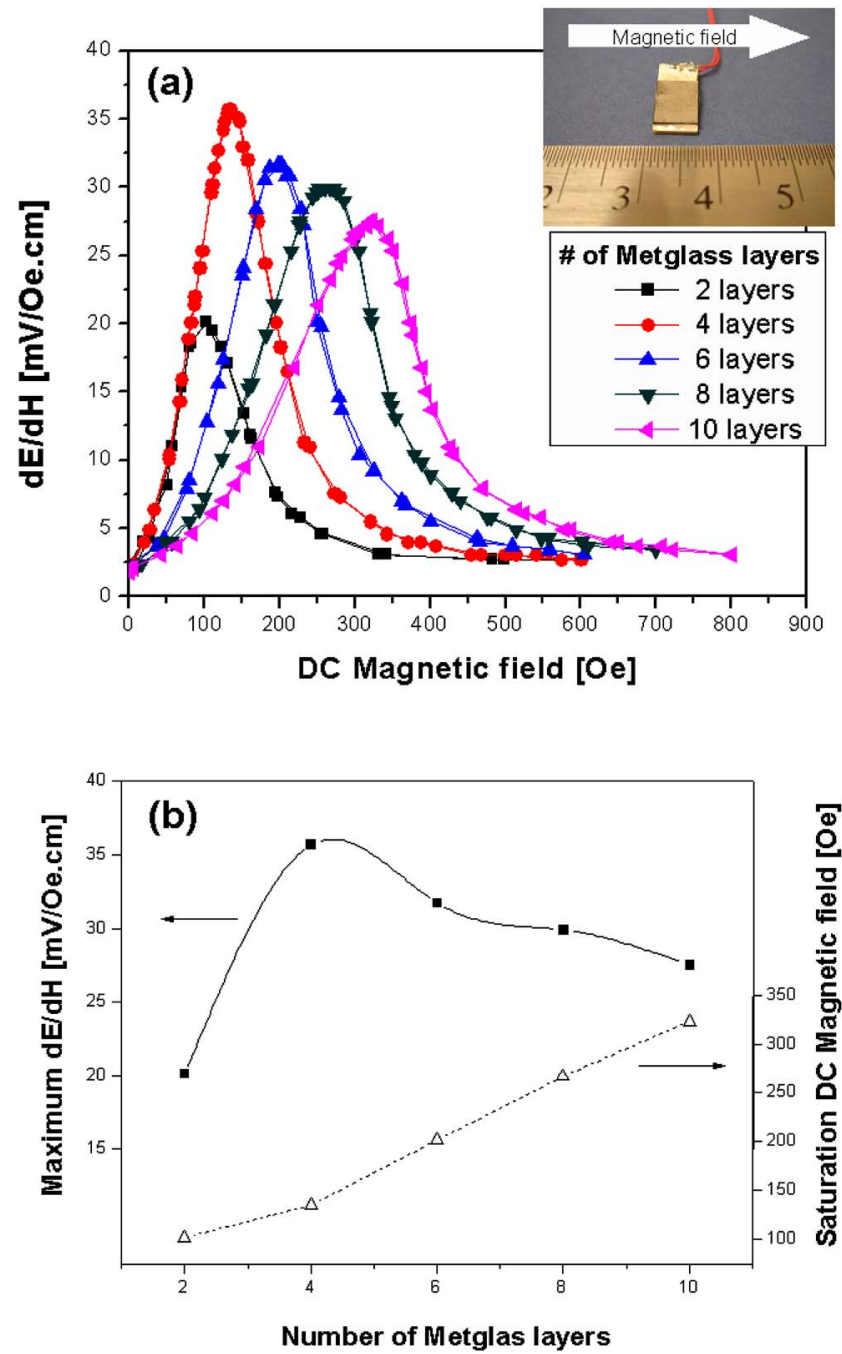

FIG. 2. (Color online) (a) ME coefficient as a function of the number of Metglas layers and dc magnetic field. (b) Maximum ME coefficient and saturation dc magnetic field as a function of the number of Metglas layers on PZNT. All measurements were conducted under $H_{\mathrm{ac}}=1$ Oe and frequency of $1 \mathrm{kHz}$.

$\times 15.5 \mathrm{~mm}^{2}$ ) (type I, unimorph). For the PZNT disk with diameter of $10.2 \mathrm{~mm}, 20$ layers of Metglas were stacked on both sides (type II, sandwich). Figure 1(b) shows the ME responses of both composite structures measured under $H_{\text {ac }}$ $=1 \mathrm{Oe}$ at $1 \mathrm{kHz}$. Type I exhibited maximum output voltage of $62 \mathrm{mV} / \mathrm{cm}$ Oe at $215 \mathrm{Oe}$, while type II showed ME coefficient of $73 \mathrm{mV} / \mathrm{cm}$ Oe at $570 \mathrm{Oe}$. It is interesting to note that by changing the structure from "unimorph" type to "sandwich" and from square shape to disk, the maximum in ME coefficient was shifted by more than two times (355 Oe shift). This brings three interesting questions into picture. (i) What is the effect of stacking configurations on the maximum position of ME coefficient? (ii) How can the two configurations be combined such that ME coefficients are at maximum magnitude over the dc bias range of 215-570 Oe? (iii) What is the effect of interface area on ME coefficient? We answer these questions in the next few sections.

\section{B. Effect of number of Metglas layers}

In order to investigate the role of the number of Metglas layers on ME response, unimorph configuration was selected 
(a)
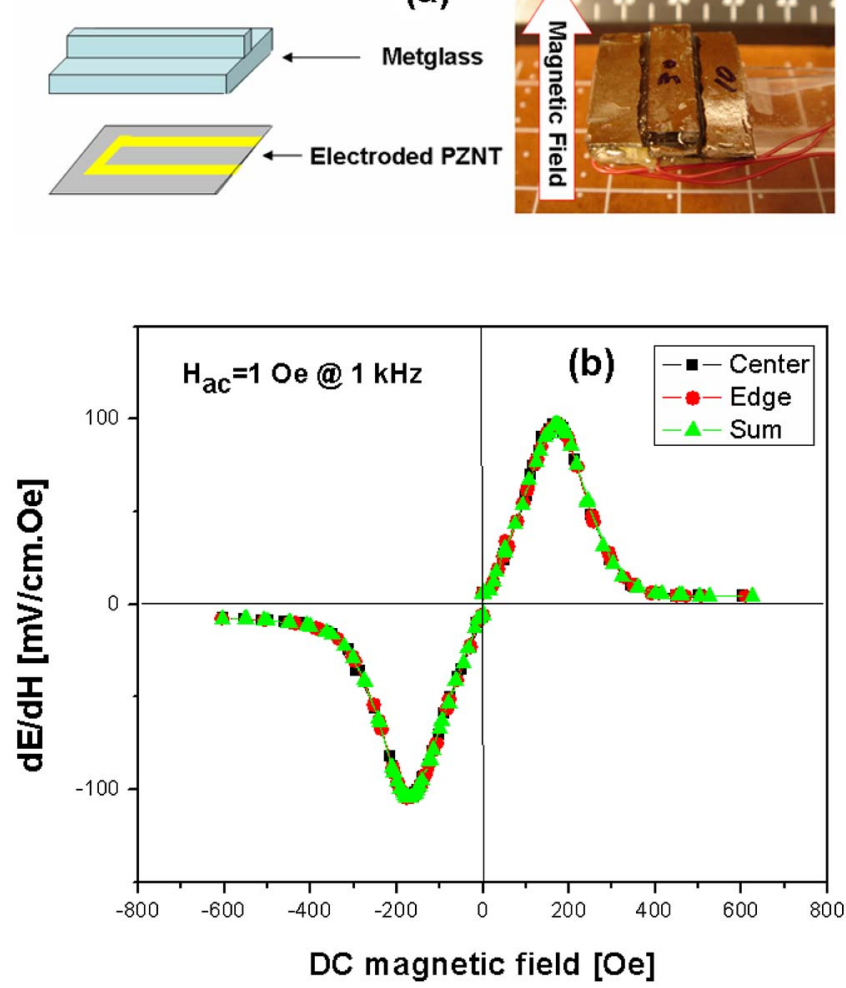

FIG. 3. (Color online) (a) Schematic representation of sample preparation with pyramid Metglas structure on separated electrodes. (b) ME output voltage as a function of applied dc magnetic field.

and two layers of Metglas with dimension of $7 \times 9 \mathrm{~mm}^{2}$ were incrementally stacked on PZNT plate of $7 \times 15 \mathrm{~mm}^{2}$. Figure 2(a) shows the variation in ME output voltage as a function of the number of Metglas layers and applied dc magnetic field. The composite with four layers of Metglas exhibited maximum magnitude of ME coefficient of the order of $36 \mathrm{mV} / \mathrm{cm}$ Oe. With further increase in the number of Metglas layers, the ME output steadily decreases. The optimum magnitude of magnetic dc bias (saturation dc magnetic field for magnetostriction coefficient) was found to increase with an increase in the number of Metglas layers. Figure 2(b) summarizes the changes in ME coefficient and saturation dc magnetic field as a function of number of Metglas layers. These results indicate that as the number of Metglas layers increases, higher dc bias is required to reach the maximum $\mathrm{ME}$ coefficient. It means that it is possible to control the ME output value and saturation point by changing the number of Metglas layers.

\section{Effect of elastic coupling on electrode structure}

In order to gain more insight into elastic coupling processes of unimorph configuration, a sectioned electrode pattern was designed on PZNT plate of $15.5 \times 15.5 \mathrm{~mm}^{2}$. As shown in the schematic in Fig. 3(a), the electrode pattern on top of the PZNT plate is divided into two parts, a stripe in between the "U" structure. The gap between the two sections was of the order of $1 \mathrm{~mm}$. Ten layers of Metglas were attached on PZNT covering the whole surface area. In the center, on top of the stripe electrode, the number of Metglas
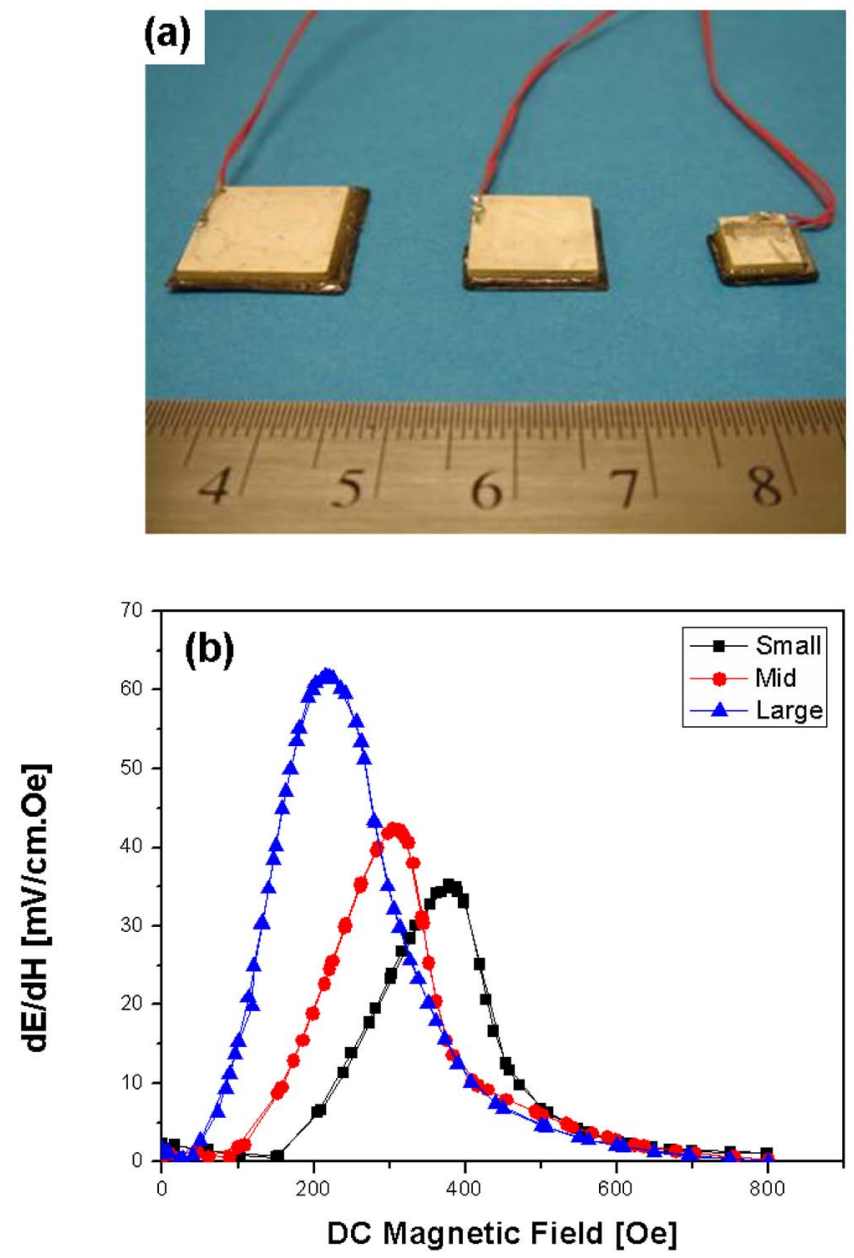

FIG. 4. (Color online) (a) Synthesized composites for understanding the effect of dimensions. 20 layers of Metglas were attached on all three PZNT plates. (b) ME output voltage variation as a function of dc magnetic field for various dimensions. All ME measurements were conducted under $H_{\mathrm{ac}}$ $=1$ Oe at $1 \mathrm{kHz}$

layers was increased to 30 layers as shown in Fig. 3(a). ME coefficients were measured from the two sections separately and then combined response from two sections was measured, as shown in Fig. 3(b). Although locally different ME output behavior was expected from each section because of the difference in number of Metglas layers, the magnitudes of ME coefficient and saturation dc magnetic field were found to be identical from both sections. This indicates that average strain distribution is homogeneous, even though locally different strains are generated. Thus, it is important to have a larger physical separation in order to achieve differences between the peak positions of ME coefficient from two separated sections.

\section{Effect of planar dimensions on ME composite in LT mode}

PZNT plates with different planar dimensions (15.5 $\times 15.5,12.5 \times 12.5$, and $8 \times 8 \mathrm{~mm}^{2}$ ) but same thickness of 1 $\mathrm{mm}$ were used in this experiment. 20 layers of Metglas were attached on each plate, as shown in Fig. 4(a) (unimorph configuration). Higher number of Metglas layers was used to avoid dependency on number of Metglas layers [saturated 
(a)
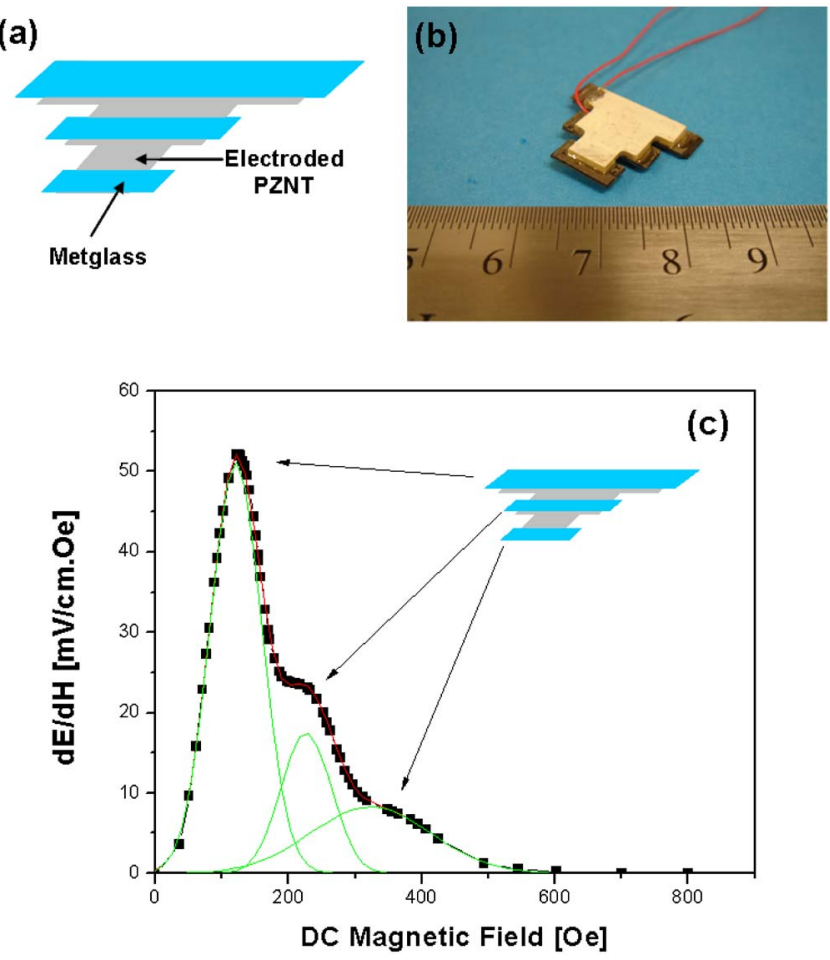

FIG. 5. (Color online) (a) Schematic structure of the dimensionally gradient sample. (b) Picture of the fabricated sample. 20-layers of Metglas was attached on each rectangular section. (b) ME coefficient of the dimensionally gradient sample under $H_{\mathrm{ac}}=1$ Oe at $1 \mathrm{kHz}$.

response in Fig. 2(b)]; therefore, the ME coefficient is dependent only on the planar dimensions of the composite. Figure 4(b) shows the ME behavior as a function of dc magnetic field for three different samples. It was found that as the planar area of composites decreases, the ME voltage coefficient decreases, while saturation dc magnetic field increases. Thus, ME coefficient and saturation dc magnetic field exhibit inverse behavior with increasing planar area. In other words, when the planar geometry is large, ME coefficient is also large at smaller magnetic dc bias. The results of Fig. 4 can be explained on the basis of edge effect called "shear lagging." As dimensions of ME composites become smaller the relative surface and edge areas increase, leading to shear lagging.

This result is important to realize different peak positions of ME coefficient from different sections. In combination with the result in Fig. 2, it is quite interesting to note that the optimum number of layers of Metglas on piezoelectric phase with large planar dimensions can provide higher ME coefficient at smaller magnetic dc bias. These results (Figs. 2 and 4) lead us to the design rule that maximum peak of the ME coefficient can be shifted by altering the geometry of laminate composites and by designing structure with gradient dimensions.

\section{E. Effect of dimension gradient on laminate composite}

In order to achieve the individual response from separate sections of composite, a new geometrical structure was conceived. The composite design was guided by the results of
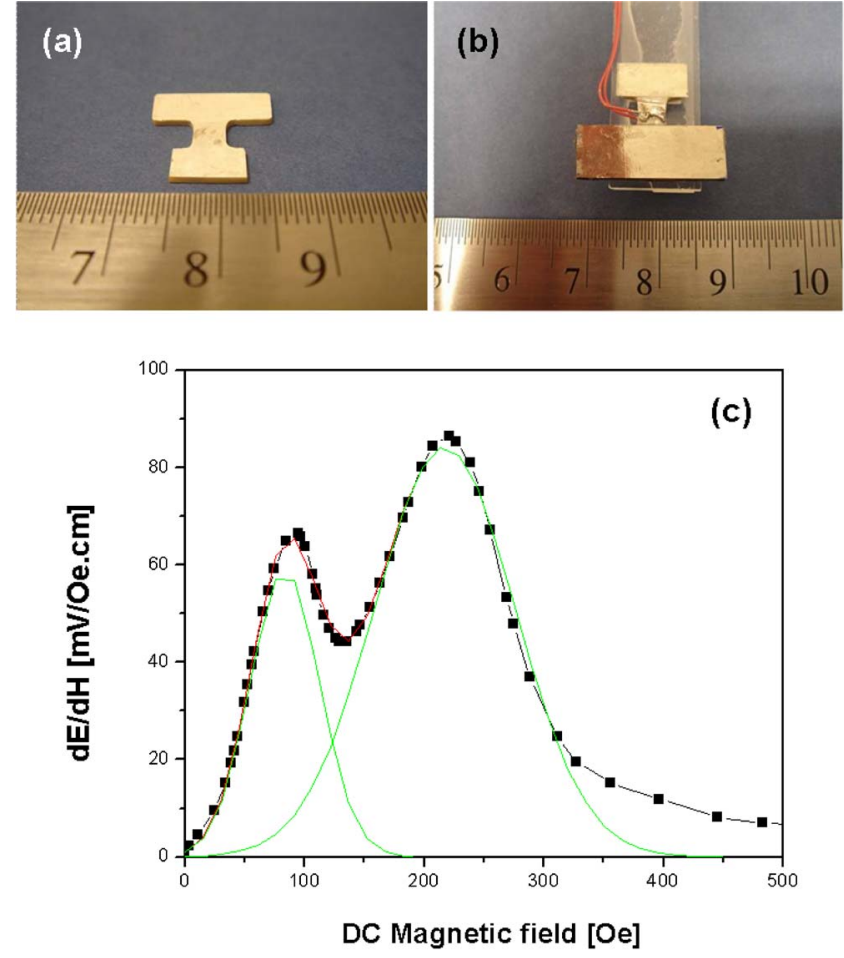

FIG. 6. (Color online) (a) Machined and electroded H-shaped PZNT plate. (b) Metglas-attached on the PZNT on both rectangular sections. (c) Broadband $\mathrm{ME}$ behavior of the $\mathrm{H}$-shaped laminate.

previous sections that (i) different dimensions resulted in different peak positions with respect to magnetic dc bias and (ii) physical separation between the Metglas layers was necessary to achieve difference in the peak positions of ME coefficient. Therefore, we incorporated these two factors by designing a dimensionally gradient composite with separate Metglas sections, as shown in Fig. 5(a). The dimensions of the PZNT plate in each section were as follows: $15.5 \times 5$, $10.6 \times 5.5$, and $6.2 \times 5 \mathrm{~mm}^{2}$ (width of about $5 \mathrm{~mm}$ was reduced per $5 \mathrm{~mm}$ length) as shown in Fig. 5(b). The thickness of the plate was $1 \mathrm{~mm}$. On this structure, 20 layers of Met-

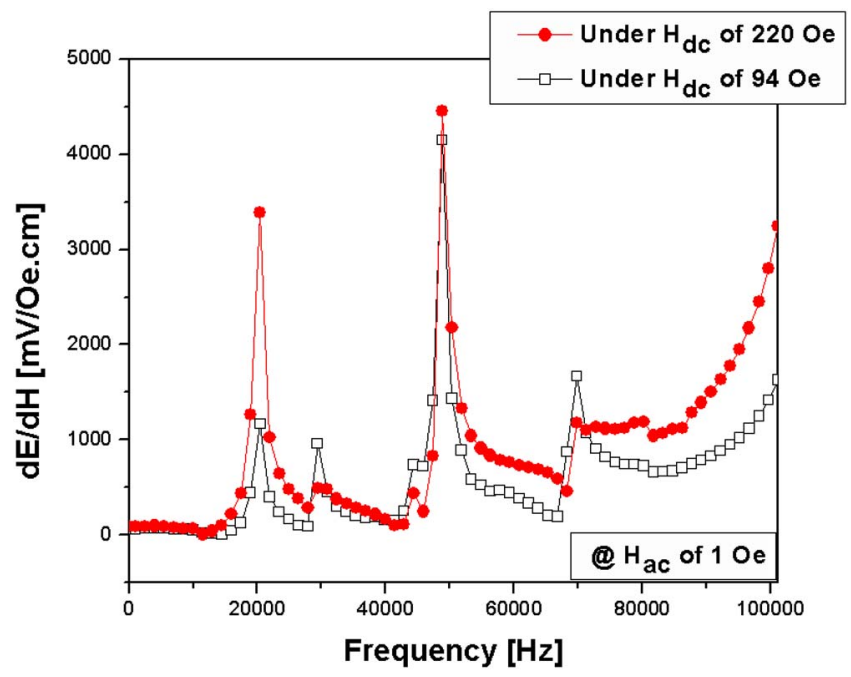

FIG. 7. (Color online) Frequency dependence of the ME coefficient for broadband laminate composite. Measurements were conducted under $H_{\mathrm{ac}}$ $=1$ Oe and $H_{\mathrm{dc}}=94$ and 220 Oe. 


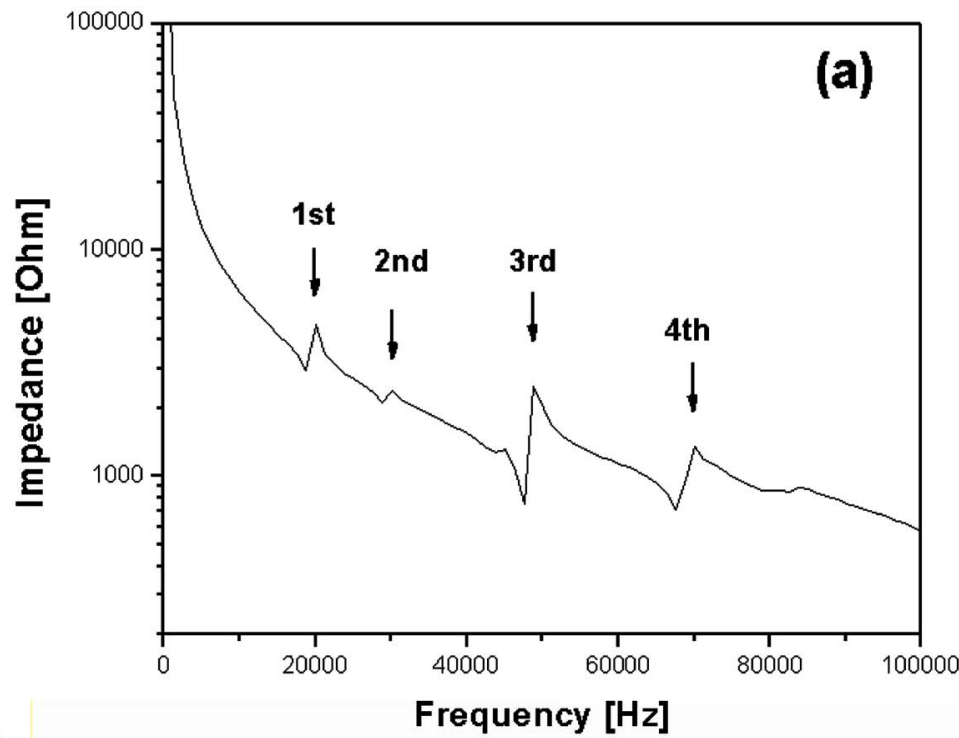

(b)

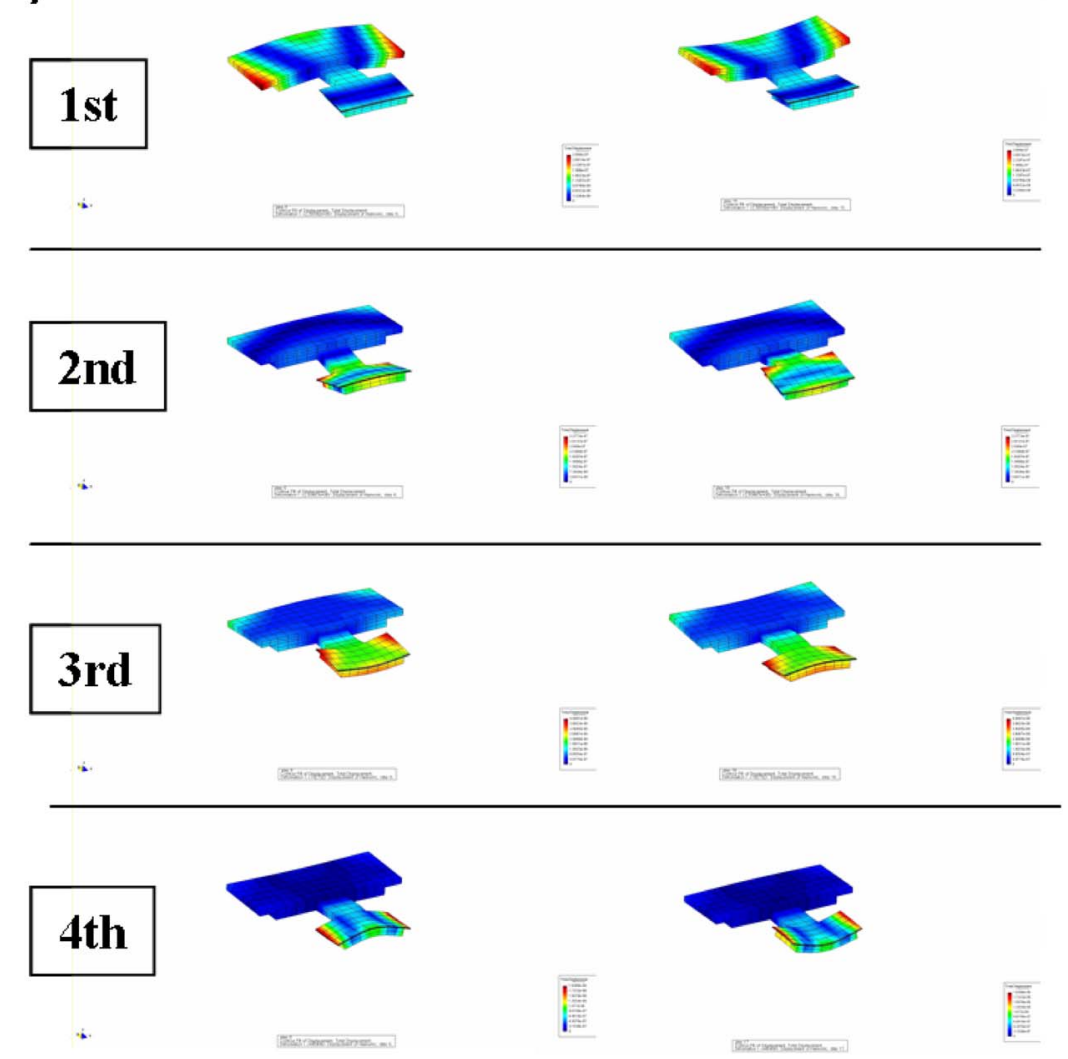

FIG. 8. (Color online) Resonance analysis. (a) Impedance spectrum. (b) FEM analysis of bending resonance modes. The peak positions are indicated in (a). glas with proportional surface area were attached as shown in Fig. 5(b), where each Metglas section was separated from each other by $1.5 \mathrm{~mm}$. We intentionally selected 20 layers of Metglas so that the observed effect is solely from the variation in the dimension across the composite. Figure 5(c) shows the measured ME response of this composite structure. Interestingly, total ME response of the structure was in combination of the three individual effects, as shown by the peak fitting: three typical components were found in the same specimen. As the planar dimension of composite decreases, the saturation magnetic field increases in providing three separate peaks from three different sections. The larger composite section exhibits higher ME coefficient at smaller magnetic dc bias, in agreement with our result in Fig. 4.
The results in Figs. 3-5 lead us to an important conclusion that high magnitude and saturation point of ME coefficient can be controlled by composite structure having separated sections, dimensions of composite, and number of Metglas layers on a PZNT planar area. Based on this conclusion, we tested and demonstrated the broadband ME sensor in the next section.

\section{F. Observation of broadband behavior}

An asymmetric $\mathrm{H}$ shape PZNT plate was fabricated with the dimensions shown in Fig. 6(a) $(15.2 \times 5.3$ and 8.5 $\times 5 \mathrm{~mm}^{2}$ areas were connected with the bridge of 4 $\times 5 \mathrm{~mm}^{2}$ ) in order to enhance physical separation. In this 
design, the bridge connecting two different dimensions of laminate composites will lead to two separate responses. On this PZNT plate, four layers of Metglas were attached for smaller area, and 30 layers of Metglas were attached on the larger area. It should be noted here that smaller dimensions lead to smaller ME coefficient, but smaller number of Metglas layers leads to larger ME coefficient. Thus, a compromise between the two opposing effects was calculated to be in the range equivalent to that of the large composite with 30-layered Metglas. Figure 6(b) shows the picture of the fabricated composite.

Figure 6(c) shows the measured ME response from this composite structure. The first peak of ME coefficient was found at 94 Oe associated with that of four-layered Metglas on smaller piezoelectric surface area. The second peak of ME coefficient was found at 220 Oe resulting from the 30layered Metglas on the larger piezoelectric area. There is a slight drop in the magnitude of ME coefficient between the two peaks, which reflects a mismatch in tailoring the dimensions of two sections. By further adjusting the dimensions, it is possible to bring the two peaks closer to each other and achieve almost a square wave response. However, the results of this figure clearly demonstrate the idea in designing the broadband ME sensor.

\section{G. Frequency dependency of broadband ME sensor}

Figure 7 shows the frequency dependence of ME coefficient for broadband composite in the range from 40 to $10^{5} \mathrm{~Hz}$. Measurement was conducted at $H_{\mathrm{ac}}=1$ Oe under applied dc magnetic fields of 94 and 220 Oe. The peaks in this figure correspond to electromechanical resonances occurring at 20,29, 49, and $70 \mathrm{kHz}$. It is interesting to note that regardless of the applied dc magnetic field in the range of 94-220 Oe, the broadband ME sensor showed similar range of ME output voltage of $\sim 4 \mathrm{~V} / \mathrm{cm}$ Oe at $49 \mathrm{kHz}$. The maximum ME output was $4.5 \mathrm{~V} / \mathrm{cm}$ Oe under the conditions of $H_{\mathrm{ac}}=1$ Oe at $49 \mathrm{kHz}$ and $H_{\mathrm{dc}}=220$ Oe.

In order to find the electromechanical resonance modes of PZNT plate-Metglas laminate, a FEM analysis was conducted using ATILA. Figure 8(a) shows the impedance spectrum for $\mathrm{H}$-shaped broadband laminate. The impedance spectrum was found to exhibit resonance peaks at the same position as that observed in ME measurements as a function of frequency. This further indicates that all the peaks were correlated with electromechanical resonance modes. Figure 8(b) shows the bending oscillation modes for the four resonances pointed in the impedance spectrum. The first mode at $20 \mathrm{kHz}$ was found to be from bending of the large rectangular section in the H-laminate [Fig. 8(b), first]. The second mode at $29 \mathrm{kHz}$ was found to be from the bending of bridge structure joining the two rectangular sections [Fig. 8(b), second]. The output voltages from second mode were relatively low, which could be associated to the small deformation occurring in the bridge. The third mode at $49 \mathrm{kHz}$ was related to combined bending response from bridge and small rectangular section of the composite [Fig. 8(b), third]. This mode generated the highest output voltages as shown in Fig. 7. The fourth mode at $70 \mathrm{kHz}$ was found to be related to bending of small rectangular section of composite [Fig. 8(b), fourth]. The results indicate that third resonance mode at $49 \mathrm{kHz}$ is combination of second mode occurring at $29 \mathrm{kHz}$ and fourth mode occurring at $70 \mathrm{kHz}$ and leads to larger displacements in the structure, resulting in higher ME coefficient. These results further provide the insight in designing a composite structure that could provide large ME coefficient over a wide frequency range.

\section{CONCLUSIONS}

In this study, we demonstrate a broadband magnetoelectric composite using Metglas and PZNT plates. Various structural and geometrical parameters were investigated to understand the variation in ME coefficient with applied dc magnetic bias. By controlling laminate dimensions, number of Metglas layers, and gradient geometry, we found the correlation between the overall deformation and peak position in ME coefficient and used it to demonstrate a working prototype of the sensor.

\section{ACKNOWLEDGMENTS}

This work was financially supported by Army Research Office, USA, (Grant No. 47576MS) and Office of Basic Energy Science, Department of Energy, USA (Grant No. DEFG02-08ER46484). The authors from KIMS acknowledge the funding through Component-Material Development Program, Ministry of Knowledge Economy, Republic of Korea.

${ }^{1}$ N. A. Spaldin and M. Fiebig, Science 309, 391 (2005).

${ }^{2}$ Y. Lin, N. Cai, J. Zhai, G. Liu, and C.-W. Nan, Phys. Rev. B 72, 012405 (2005).

${ }^{3}$ D. V. Chashin, Y. K. Fetisov, K. E. Kamentsev, and G. Srinivasan, Appl. Phys. Lett. 92, 102511 (2008).

${ }^{4}$ J. Ryu, A. Vazquez Carazo, K. Uchino, and H.-E. Kim, J. Electroceram. 7, 17 (2001).

${ }^{5}$ M. Avellaneda and G. Harshe, J. Intell. Mater. Syst. Struct. 5, 501 (1994).

${ }^{6}$ G. Shrinivasan, E. T. Rasmussen, B. J. Levin, and R. Hayes, Phys. Rev. B 65, 134402 (2002).

${ }^{7}$ J. Ryu, A. V. Carazo, K. Uchino, and H.-E. Kim, Jpn. J. Appl. Phys., Part 1 40, 4948 (2001).

${ }^{8}$ S. Dong, J.-F. Li, and D. Viehland, J. Appl. Phys. 95, 2625 (2004).

${ }^{9}$ S. Dong, J. Zhai, J.-F. Li, and D. Viehland, Appl. Phys. Lett. 89, 252904 (2006).

${ }^{10}$ R. A. Islam and S. Priya, J. Mater. Sci. 43, 2072 (2008).

${ }^{11} \mathrm{~S}$. Dong, J.-F. Li, and D. Viehland, IEEE Trans. Ultrason. Ferroelectr. Freq. Control 50, 1253 (2003).

${ }^{12}$ S. Dong, J. Cheng, J.-F. Li, and D. Viehland, Appl. Phys. Lett. 83, 4812 (2003).

${ }^{13}$ S. Dong, J.-F. Li, and D. Viehland, J. Mater. Sci. 41, 97 (2006).

${ }^{14}$ C.-M. Chang and G. P. Carman, Phys. Rev. B 76, 134116 (2007). 